\title{
THE ROLE AND RESPONSIBILITY OF CLINICAL PHARMACISTS IN RHEUMATOLOGY CLINIC: AN EXPLORATORY STUDY IN HONG KONG.
}

\author{
Shirley Wai Ying YIPl, Gary Chung Hong CHONG ${ }^{1}$
}

1. United Christian Hospital, Hospital Authority, Hong Kong

Correspondence: ywy510@ha.org.hk

\begin{abstract}
In Hong Kong, arthritis is the third leading chronic disorder among the older people. It could create a huge amount of burden on the health care system if patients cannot achieve good diseases control and have recurrent flare up of diseases. To maintain stable control and minimize acute flare up, medication adherence is essential. Studies revealed that drug education has the highest evidence in improving medication adherence. Pharmacists who are expert in drugs can provide counseling to arthritis patients, improving their drug adherence and disease activities. As a result, research evaluating the effectiveness of pharmacist counseling service on improving arthritis patients' medication adherence and disease activities in Hong Kong has been initiated in a specialist out-patient clinic of a local acute hospital.
\end{abstract}

This research aims to improve patient medication adherence and enhance medication safety. A validated Compliance Questionnaire on Rheumatology (CQR-19) is used to measure the medication adherence. From the preliminary data, over $90 \%$ of the recruited subjects are non-adherence at baseline and thus, detailed drug counseling is necessary.

During the first visit, pharmacists will provide a 20 minutes drug counseling service to the referred patients. This includes discussion on drug administration schedule, importance of drug adherence, side effects management and pain management. Besides, pharmacists also have different roles in other aspects such as drug information and procurement. Pharmacists would assist in providing evidence-based information and recommendations to physicians and nurses for drug-related enquiries. These attempts to improve therapeutic outcomes and minimize medication errors, enhance medication safety and reduce hospitalization. Design of the multidisciplinary care model and results of this study would provide a reference for the future development of clinical pharmacist service in rheumatology.

\section{KEYWORDS}

clinical pharmacist, rheumatology, arthritis, medication adherence, medication safety, DMARDs

\section{INTRODUCTION}

In Hong Kong, arthritis is a highly prevalent condition among older people and can cause joint deformation and long-term disability, leading to reduced quality of life. According to a study conducted by Census \& Statistics Department on older persons aged over 60 years old in domestic households, arthritis is the third leading chronic disorder among them with prevalence of $19.9 \%$ in an estimated 795800 elderly with chronic disease population. [1] According to the National Institutes of Health (NIH) of the United States, arthritis is a disease condition instead of a single disease. It refers to joint inflammation which usually leads to joint pain, swelling of joints and stiffness of joints. [2] 
There are different types of arthritis such as rheumatoid arthritis (RA), ankylosing spondylitis (AS) and psoriatic arthritis (PSA). These arthritis diseases are also the comorbidities of other rheumatic diseases such as myositis. From the data of a hospital based cross-sectional retrospective study, the estimated average total costs for management of a type of arthritis, RA, in Hong Kong during 2006-2007, were already $\$ 9286$ US dollar per patient year. [3] Due to the high prevalence rate of arthritis in elderly, it could be expected that huge amount of burden would be created on the Hong Kong health care system if patients' diseases are not under good control.

Satisfactory medication adherence is essential in achieving good disease control. According to several studies in RA patients, higher medication adherence is statistically significant associated with lower flare rate and disease activity score (DAS-28). [4, 5] Effective disease control at an early stage can also prevent irreversible joint damage and hence improve patients' quality of life. [4] Study data also supported that drug counseling and education has the highest evidence in improving medication adherence. [6] Pharmacists who are the experts in drugs are capable of providing counseling to arthritis patients, improving their drug adherence and disease activities. Therefore, research evaluating the effectiveness of pharmacist counseling service on improving arthritis patient's medication adherence and disease activities in Hong Kong is initiated in a specialist out-patient clinic of United Christian hospital.

In this paper, the role and responsibility of clinical pharmacists in rheumatology clinic of Hong Kong will be discussed from various aspects: 1) multidisciplinary care model in rheumatology clinic 2) role in patient counseling and collaboration with other health care professionals, and 3) Benefits of clinical pharmacists in rheumatology clinic.

\section{MULTIDISCIPLINARY CARE IN RHEUMATOLOGY CLINIC}

Clinical pharmacist services have been well established in many specialties such as oncology, pediatric and geriatric to promote medication adherence and enhance medication safety. Studies have illustrated that clinical pharmacists significantly reduce medication errors and improve patients' medication adherence through drug education. [7-10] Clinical pharmacists also have close collaboration with other health care professionals such as physicians and nurses, providing evidence-based recommendation to optimize drug regimens and treatment outcomes. $[8,9,11]$ Therefore, it is worthwhile to conduct this research to evaluate the effectiveness of clinical pharmacist counseling service in rheumatology.

In a few countries, pilot pharmacist-led rheumatologic clinics has been set up for patient counseling although the study data is limited.

In Canada, an observational study comparing patient satisfaction between the pharmacist physician collaborative care model and traditional physician provided care in rheumatology clinic occurred in 2015. [12] Pharmacists provided patient education and shared decision making on treatment regimens.

From the study results, patients showed statistically significant higher mean overall satisfaction score in the pharmacist physician collaborative care model (collaborative care: 4.56; physician: 4.30; $\mathrm{P}=0.02$ ). [12] Patients also showed higher general satisfaction (collaborative: 4.61; physician: 4.25; $\mathrm{P}=0.005$ ) and satisfaction in information provided (collaborative: 4.53; physician: $4.20 ; \mathrm{P}=0.007$ ) in the collaborative care model. [12] In addition, the results also verified that the clinic capacity was increased by $50 \%$ in the collaborative care model [12] and therefore more patients could be seen by the rheumatologists in each session. This reduces the waiting time of new cases.

Another 6-month study was undertaken in Singapore in 2010 which measured patient satisfaction of a pharmacist/advanced practice nurse (APN)-led rheumatology monitoring clinic. [13] In the study, patients were reviewed by rheumatologist and pharmacist/APN at alternative intervals. There were four pharmacists and two APN involved in the service. (13) In this monitoring clinic, the pharmacist/APN would review the effectiveness of the treatment and monitor for adverse drug reactions. If the patients were stable, a repeated prescription would be issued to them. If drug-related problems or disease flare was detected, patients would be referred to a rheumatologist immediately. [13] Over $90 \%$ of the patients were satisfied with the monitoring clinic as they could understand the disease better. The patients were also more likely to adhere to the treatment. [13] On the other hand, over $80 \%$ of rheumatologists agreed that this service saved their time which allowed them to focus on complex cases and improve overall patient care. [13] 
These study results demonstrated that clinical pharmacists service in rheumatology can enhance patient care and patients' disease knowledge, leading to improvement in medication adherence. It can also allow more time for rheumatologist to assess patients. However, there is a lack of studies focused on measuring improvement in patient medication adherence and disease activity after receiving pharmacist counseling in rheumatology.

This research attempts to evaluate the effectiveness of pharmacist counseling service on improving arthritis patient's medication adherence and disease activities in Hong Kong. Since rheumatology includes a large variety of diseases, this research only focuses on arthritis patients, mainly rheumatoid arthritis (RA), ankylosing spondylitis (AS) and psoriatic arthritis (PSA) patients.

The aims of this research are to 1) improve patient medication adherence 2) identify drug-related problems or adverse drug reactions 3) enhance medication safety and, 4) improve patient disease activity.

A multidisciplinary care model including physicians, nurses and pharmacists have been designed. Physicians and rheumatology nurses will refer new arthritis patients, patients with complex regimens and patients with nonadherence issues to this study. Referred patients who are on at least one oral disease-modifying antirheumatic drugs (DMARDs) or steroid will be recruited. Upon completion of the first pharmacist counseling visit, a phone follow-up at week 6 and a face-to-face follow-up visit at week 12 will be arranged for the recruited subjects.

During the initiation assessment, baseline medication adherence of the recruited subjects will be measured with the Compliance Questionnaire on Rheumatology (CQR19). This questionnaire is validated in rheumatology for measuring patient medication adherence. [14] The total score ranges from 0-100 and a higher score indicates a higher level of medication adherence. According to the validation studies, patients with CQR-19 score greater than 80 are indicated as adherence while those less than or equal to 80 are indicated as non-adherence. [14] The mean score and the number of adherence patients before and after pharmacist counseling will be compared in the study.

The improvement in disease activity of recruited subjects and its association with the improvement in medication adherence are the secondary outcomes. The disease activity of RA, PsA and most other arthritis patients will be measured by disease activity score (DAS-28) while that for AS patients will be measured by Bath Ankylosing Spondylitis Disease Activity Index (BASDAI).

From the data collected till 1/2020, the medication adherence of the recruited subjects is not desirable. Over $90 \%$ of the recruited subjects are non-adherence with baseline CQR-19 score not more than 80. Since the administration schedule of some DMARDs are not at daily basis such as methotrexate (MTX) is usually prescribed as once weekly dosing, patients would miss the doses easily especially during initiation of new treatment regimens. During phone follow up discussion with patients, adverse drug reaction or drug-related problems are identified in one third of the recruited subjects. These preliminary data justified that detailed drug education and counseling is necessary for improving medication adherence in arthritis patients.

\section{ROLE OF CLINICAL PHARMACISTS IN RHEUMATOLOGY CLINIC}

Pharmacists are well-educated in drug therapies and thus competent to provide drug counseling to arthritis patients. Besides patient education, pharmacists also have distinctive roles in other aspects such as dispensing and drug procurement. These will be discussed below.

\section{PATIENT EDUCATION}

Patient education includes discussion on drug administration schedule, importance of drug adherence, side effects management and pain management. Pharmacists will provide a 20 minutes drug counseling service to the referred patients during the first visit. According to World Health Organization (WHO), medication adherence refers to the extent to which a person's behavior corresponds with agreed recommendations from a health-care provider which means taking the right medications at the right time, dosage, and frequency. [15] Therefore, educating patients on correct drug regimens are vital. Since DMARDs and prednisolone used for disease control of arthritis usually require escalating and tapering regimen respectively, drug calendars specified the date of dose increment or reduction will be prepared and explained in detail to patients. Chinese drug name labels of DMARDs will be provided to facilitate the communication with patients. In addition, pharmacists will discuss the drug-drug and drugfood interactions with patients and provide 
recommendation on the management. For example, cyclosporine A (CSA) which is frequently used for treatment of PSA will interact with grapefruit juice, leading to an increase in plasma concentration of CSA and hence increasing the risk of toxicity. Therefore, pharmacists are to remind those patients that they have to avoid grapefruit juice all the time. Besides, use of Chinese herbal medicine such as Lingzhi and Ginseng are very popular in Hong Kong and Asian countries. However, these herbal medicines usually stimulate the immune system. Since arthritis are autoimmune disorder that joints are attacked by patients own immune system, these herbal medicines may worsen their disease control. Furthermore, concomitant use of DMARDs and herbal medicines could easily lead to derangement of liver function test. Hepatotoxicity is a side effect of many DMARDs such as MTX and Leflunomide while herbal medicines are commonly metabolized by liver enzymes. As a result, this increases the risk of DMARDs induced hepatotoxicity and may interrupt the treatment schedule, worsening the disease control of patients. Therefore, pharmacists will evaluate the use of Chinese herbal medicine and supplements in arthritis patients and provide individualized recommendation on the management during the counseling.

Besides education on drug regimens, disease knowledge education and understanding patients' belief towards the treatment are crucial in improving adherence. According to WHO reports, there are many factors affecting patient medication adherence. The immediacy of beneficial effects is one of them while DMARDs usually need to take 1-2 months to achieve their effects. [15] Consequently, pharmacists will discuss their slow onset of action and confer the long-term consequence for uncontrolled diseases such as irreversible joint erosion and deformation to patients. Hence, this would encourage patients to adhere to the treatment and have good disease control at an earlier stage, thus preventing further joint damage and reduction in self-care ability.

Experience of side effects is another factor leading to drug non-adherence issue. [15] During counseling, pharmacists will explain the side effects of DMARDs and warning labels will be provided to remind patients. Advise on selfmonitoring and management skills for mild side effects will be provided to relieve patient's concerns. For example, gastrointestinal tract discomfort is one of the common side effects for hydroxychloroquine (HCQ), pharmacists would recommend patients to administer it with food to minimize the side effects. Phone follow-up is also arranged for each patient to closely monitor for and detect any possible adverse drug reactions or acute flare up of disease. If they are detected during phone follow up, recommendation on self-management will be provided and may refer to rheumatologists if necessary.

Last but not least, pharmacists will discuss both pharmacological and non-pharmacological methods for pain management. Safe use of non-steroidal antiinflammatory drugs (NSAIDs) and low dose steroid will be reviewed and discussed with patients as physicians usually allow patients to titrate these drugs according to their needs. Moreover, pharmacists will educate patients to use non-pharmacological methods such as warm and cold compress in different situations for pain relief.

Through the patient counseling, we hope to improve drug adherence of patients and enhance medication safety.

\section{VERIFICATION AND DISPENSING}

Before patients can receive their medication, their prescriptions will be screened and verified by pharmacists to prevent prescribing errors such as incorrect frequency or duration. Besides, patient's on-hand medication prescribed by other specialties will be reviewed to avoid drug interaction and duplication especially for NSAIDs. If drug-drug interaction is identified, pharmacists will provide recommendation to physicians and work out the solution with them. Afterwards, drugs will be dispensed to patients.

\section{DRUG INFORMATION AND PROCUREMENT}

Besides dispensing and patient education, clinical pharmacists would collaborate with other health care professionals including physicians and nurses to optimize patient care and treatment plans. Pharmacists would assist in providing drug information and the preparation of education materials. For example, drug sample books displayed most of the available DMARDs, NSAIDs and steroids with different strengths were prepared to facilitate the communication between physicians and patients during consultation. On the other hand, pharmacists also help in providing evidence-based information and advice to physicians and nurses for drug-related enquiries. Pharmacists are familiar with different drug search databases for solving different types of drug-related problems. They also trained for selecting and analyzing data from clinical studies and databases critically in order to formulate the most appropriate approach. Hence, they can provide recommendations with evidence support efficiently. 
In addition, procurement is fundamental to maintain stable drug supply and introduction of new drugs. When there is interruption or discontinuation of existing drugs, clinical pharmacists could immediately deliver this information to physicians and provide alternatives. When there are new treatment options available, pharmacists would help in the application process and prepare supporting data on efficacy and safety of new drugs. Hence, it can smooth the introduction of new drugs in the hospital and hence patients could have more treatment choices especially for refractory cases.

\section{THE BENEFITS OF CLINICAL PHARMACISTS IN RHEUMATOLOGY CLINIC}

Clinical pharmacists pose positive impacts on several areas in rheumatology including patients' treatment outcome, medication safety and medical expenditure.

Through pharmacist drug counseling, the tapering or escalating drug schedule can be clearly explained to patients, minimizing the risk of wrong dosage or frequency. Although dispensing labels with administration instruction are affixed on each prescribed drug, medication errors could still exist by cause of misinterpretation of drug information on the labels. [16] For example, initiation of MTX treatment often requires escalating schedule and the dose is increased every 1-2 weeks. Before the pharmacist counseling service, considerable number of patients could not follow the schedule. They may wrongly administer MTX every day but it should be taken once weekly, leading to overdose and toxicity. Some patients could not step up MTX to targeted dosage due to misunderstanding, leading to sub-therapeutic effects and insufficient control of the disease. After pharmacist counseling service, these situations are improved and more than $90 \%$ of the recruited patients can follow the treatment schedule.

Besides, possible drug-drug or drug-food interaction will be discussed with patients. This prevents suboptimal therapeutic effects due to reduction in drug concentration or overdose related to interaction. These can improve treatment outcome and enhance medication safety.

Moreover, pharmacists would educate patients on side effects management, preventing patients' nonadherence issue related to side effects. For examples, during initiation of treatment or switching drugs, some patients would be concerned about the side effects and do not administer the drugs as prescribed. Besides, when patients experienced side effects, most of them will stop the drugs by themselves until next follow up. After drug counseling, patients would understand the management of mild side effects and if any adverse drug reactions were detected during phone follow up, pharmacist could provide appropriate recommendation such as reducing the drugs to tolerable dosage and giving advice on pain management. This could prevent severe acute flare up of diseases due to side effects related non-adherence issue and hence improve treatment outcomes.

According to a study on RA patients in Hong Kong, more than $20 \%$ patients required accident and emergency (A\&E) visit and hospitalization due to flare of RA. [3] Some of them required hospitalization for more than one time and the mean duration of inpatient care was 5.5 days. [3] Furthermore, several studies demonstrated that pharmacist counseling reduced medication errors and hospitalization, showing net benefits in cost-saving of the medical system. [17-18] Through pharmacists counseling in rheumatology, it could improve drug adherence and disease control and enhance medication safety, reducing acute flare rate and hospitalization. Thus, it reduces the burden on and saves the medical expenditure of the public health care system.

\section{CONCLUSION}

Clinical Pharmacists in a rheumatology clinic have distinctive roles in different aspects including patient counseling, dispensing, handling drug information and procurement. They provide drug counseling to improve drug adherence and enhance medication safety. Hence, this multidisciplinary collaboration care could improve the treatment outcome and disease control of patients, further reducing acute flare up of diseases and hospitalization.

\section{Reference}

1. Hong Kong: Census and Statistics Dept. Sociodemographic Profile, Health Status and Self-care Capability of Older Persons. Thematic Household Survey Report - Report No.40. 2009.

2. National Institute of Arthritis and Musculoskeletal and Skin Diseases. Health Information on Arthritis. 2017 [cited 29 April 2019]. Available from: https://www.niams.nih.gov/health-topics/arthritis 
3. Zhu TY, Tam L-S, Li EK. Societal Costs of Rheumatoid Arthritis in Hong Kong: a Prevalence-based Cost-ofillness Study. Rheumatology. 2011;50(7):1293-301.

4. Nakagawa S, Kuwana $M$, Nakaishi $M$, Hashimoto $M$, Ito $\mathrm{H}$, Yamamoto W, et al. Effect of Medication Adherence on Disease Activity among Japanese Patients with Rheumatoid Arthritis. PLoS ONE. 2018;13(11):e0206943.

5. Li L, Cui Y, Yin R, Chen S, Zhao Q, Chen $H$, et al. Medication Adherence has an Impact on Disease Activity in Rheumatoid Arthritis: a systematic review and meta-analysis. Patient Prefer Adherence. 2017; $11: 1343-56$.

6. Lavielle $M$, Puyraimond-Zemmour D, Romand $X$, Gossec L, Senbel E, Pouplin S et al. Methods to Improve Medication Adherence in Patients with Chronic Inflammatory Rheumatic Diseases: a systematic literature review. RMD Open. 2018;4(2):e000684.

7. Whittaker C, Nelson S, Tom S. Impact of the Pharmacists' Patient Care Process on Medication Adherence in Older Adults with Multimorbidity. The Senior Care Pharmacist. 2019;34(7):456-463.

8. Chu MH., Chong $\mathrm{CH}$. Oncology Pharmacist's Role and Impact on the Multidisciplinary Patient-Centre Practice of Oncology Clinic in Public Hospitals. AsiaPacific Journal of Health Management. 2019;14(1):16.

9. Kelishadi R, Mousavinasab F. Rational Use of Medicine in the Pediatric Age Group: A summary on the role of clinical pharmacists. Journal of Research in Pharmacy Practice. 2012;1(1):10.

10. Duarte N, Barbosa C, Tavares M, Dias L, Souza R, Moriel P. Clinical Oncology Pharmacist: Effective contribution to patient safety. Journal of Oncology Pharmacy Practice. 2018;25(7):1665-1674.

11. Perlman A, Horwitz E, Hirsh-Raccah B, Aldouby-Bier G, Fisher Negev T, Hochberg-Klein S et al. Clinical Pharmacist led Hospital-wide Direct Oral Anticoagulant Stewardship Program. Israel Journal of Health Policy Research. 2019;8(1).

12. Hall JJ, Katz SJ, Cor MK. Patient Satisfaction with Pharmacist-Led Collaborative Follow-Up Care in an Ambulatory Rheumatology Clinic: Patient Satisfaction with Collaborative Care. Musculoskelet Care. 2017;15(3):186-95.

13. Chew LC, Lim TG, Loy KL, Kong MC, Chang WT, Tan SB, et al. A Questionnaire Survey of Patient Experience with the Rheumatology Monitoring Clinic in Singapore. International Journal of Rheumatic Diseases.

2012;15(4):390-8.

14. E. De Klerk, D. van der Heijde, R.Landewe, H. van der Tempel, S. van der Linden. The Compliancequestionnaire-rheumatology Compared with Electronic Medication Event Monitoring: a validation study. J Rheumatol, 30 (2003), pp. 2469-2475

15. World Health Organization. Adherence to Long-Term Therapy: Evidence for Action. 2003.

16. La Caze A. Safer Dispensing Labels for Prescription Medicines. Australian Prescriber. 2018;41 (2):46-49.

17. Imamura $M$, Ogawa D, Takatori T, Yamaguchi $M$, Takata T, Hada T et al. A Retrospective Study of the Effects of Oncology Pharmacist Participation in Treatment on Therapeutic Outcomes and Medical Costs. Biological \& Pharmaceutical Bulletin. 2017:40(11):1956-1962.

18. Najafzadeh M, Schnipper JL, Shrank WH, Kymes S, Brennan TA, Choudhry NK. Economic Value of Pharmacist-led Medication Reconciliation for Reducing Medication Errors after Hospital Discharge. Am J Manag Care. 2016;22(10):654-61. 